$12-1-1989$

\title{
Observability of Atmospheric Glories and Supernumerary Rainbows
}

James A. Lock

Cleveland State University, j.lock@csuohio.edu

Follow this and additional works at: https://engagedscholarship.csuohio.edu/sciphysics_facpub

Part of the Physics Commons

How does access to this work benefit you? Let us know!

\section{Publisher's Statement}

This paper was published in Journal of the Optical Society of America A: Optics Image Science and Vision and is made available as an electronic reprint with the permission of OSA. The paper can be found at the following URL on the OSA website: http://www.opticsinfobase.org/josaa/ abstract.cfm?URI=josaa-6-12-1924. Systematic or multiple reproduction or distribution to multiple locations via electronic or other means is prohibited and is subject to penalties under law.

\section{Original Citation}

Lock, James A. "Observability of Atmospheric Glories and Supernumerary Rainbows." Journal of the Optical Society of America A: Optics Image Science and Vision 6 (1989): 1924-1930.

\section{Repository Citation}

Lock, James A., "Observability of Atmospheric Glories and Supernumerary Rainbows" (1989). Physics Faculty Publications. 24.

https://engagedscholarship.csuohio.edu/sciphysics_facpub/24

This Article is brought to you for free and open access by the Physics Department at EngagedScholarship@CSU. It has been accepted for inclusion in Physics Faculty Publications by an authorized administrator of EngagedScholarship@CSU. For more information, please contact library.es@csuohio.edu. 


\title{
Observability of atmospheric glories and supernumerary rainbows
}

\author{
James A. Lock \\ Department of Physics, Cleveland State University, Cleveland, Ohio 44115
}

Received December 19, 1988; accepted July 7, 1989

\begin{abstract}
The finite spatial coherence width of sunlight at the Earth imposes restrictions on the production of scattering phenomena based on the interference of light waves. With the spatial coherence properties of sunlight taken into account, the visibility of the supernumerary rainbow sequence adjacent to the primary rainbow and the radii of the water droplets that produce the optimum glory intensity were calculated. A substantial reduction was found in the contrast of all the supernumeraries beyond the first few, and the peak observability of the glory occurred for water droplets with radii between 10 and $20 \mu \mathrm{m}$.
\end{abstract}

\section{INTRODUCTION}

A number of light-scattering effects that occur in the atmosphere are produced by the constructive and destructive interference of light waves. Among the most well known of these are supernumerary rainbows and the glory. In order to study these phenomena in a controlled laboratory environment, water droplets ${ }^{1,2}$ and air bubbles ${ }^{3,4}$ were illuminated with laser light, and the intensity and polarization of the resulting rainbows and glories were measured. A number of differences in the observability of these wave-interference phenomena exist when they are examined in the laboratory as opposed to when they occur naturally in the atmosphere. The observability of natural glories and supernumerary rainbows depends on many factors, which can be classed broadly as either physical or physiological. The physical factors influence the contrast of the wave-interference pattern before the scattered light enters the eye of the observer. Among these factors are surface-wave damping on the water droplet, the spatial coherence properties of sunlight, multiple scattering within the cloud or rain shower, and the distribution of the sizes of the water droplets in the atmosphere. The physiological factors include color perception and color threshold discrimination within the observer's visual system. For example, because the sun is a white-light source, to calculate the colors perceived by an observer requires that the chromaticity coordinates of the interference pattern be integrated over all the visible wavelengths present in the light scattered from a single target. ${ }^{5}$ This washes out the colors observed in a white-light interference pattern and reduces the fringe contrast perceived. ${ }^{6}$ The perceived contrast is reduced further when the chromaticity coordinates of the interference pattern are integrated over the size distribution of the scattering targets.

One of the chief physical factors influencing glory and supernumerary observability is the spatial coherence width of sunlight at the Earth. The laser light used in the laboratory has a large degree of spatial and temporal coherence. Such coherence is standardly assumed in the Mie-theory analysis of scattering phenomena. On the other hand, sunlight possesses only a small degree of spatial and temporal coherence. For a droplet of a given size, this places a limit on the number of supernumerary rainbows that can be produced adjacent to the primary rainbow. It also places a limit on the size of water droplets that can produce a glory. The purpose of this paper is to estimate these limits and to emphasize their physical interpretation.

The spatial coherence properties of sunlight can be modeled with the following simple physical picture. Consider an extended light source $S$ a distance $z_{i}$ from the scattering target. It has a radius $s$ and is composed of independent radiators. Consider a single wavelength $\lambda$ of the light emitted by the source. A wave front of light from the extended source arriving at the scattering target consists of many isoplanatic patches, with the average diameter ${ }^{7}$

$$
d \approx \frac{2 \lambda}{\pi}\left(\frac{z_{i}}{2 s}\right) .
$$

The phase of the light within each patch is constant, and it varies randomly among different patches. For the case of sunlight, $d \approx 40 \mu \mathrm{m}$ at the location of the Earth. ${ }^{8}$ When a wave front of sunlight scatters from the target, two scattered light rays arriving at the position of the observer exhibit interference if they originate from within the same isoplanatic patch on the incident wave front. They do not exhibit interference if they originate in different patches. Supernumerary rainbows and the glory may be thought of as being produced by the interference of light rays that enter the water droplet at different locations on its surface. If the distance between the incident light rays producing the supernumeraries or the glory is larger than $d$, the rays are incoherent with respect to one another, and wave interference does not occur. By quantitatively calculating the visibility of atmospheric supernumerary rainbows and the glory, one can test the validity of this simple physical picture.

The effect of an incoherent-light source on wave interference can be described quantitatively as follows. Consider one of the independent radiators within the source. If the single radiator acted as a source for diffraction or scattering from the target of radius $a$, the diffracted or scattered intensity, as a function of the coordinate $\mathbf{r}_{0}$ on the viewing screen a distance $z_{0}$ behind the target in the far scattering zone, takes the form 


$$
I_{0}\left(\mathbf{r}_{0}\right)=\frac{I_{\text {inc }}}{\lambda^{2} z_{0}^{2}} G^{2}(a) F\left(K x^{m} \frac{\mathbf{r}_{0}}{z_{0}}\right),
$$

where $K$ and $m$ are constants, $I_{\text {inc }}$ is the incident intensity, and

$$
x=\frac{2 \pi a}{\lambda}
$$

The factor $F$ in Eq. (1.2) contains the interference pattern characterizing the diffraction or scattering. The geometric scaling factor $G$ is the cross-sectional area of the region on the target surface that is pierced by the incident rays responsible for the interference. For example, if the target is a circular aperture, then $m=1, K=1$, and the wave-interference factor $F$ for the diffracted intensity is

$$
F=\frac{4 J_{1}^{2}\left(\frac{x r_{0}}{z_{0}}\right)}{\left(\frac{x r_{0}}{z_{0}}\right)^{2}} .
$$

Similarly, rays passing through the aperture at any location contribute to the interference, giving

$$
G=\pi a^{2} .
$$

If the single independent radiator is replaced by the entire incoherent extended source in the source plane having coordinates $\mathbf{r}_{i}$, the intensity on the viewing screen becomes a convolution of the scattering pattern from a single radiator with the finite extent of the incoherent source, ${ }^{9}$

$$
I_{0}\left(\mathbf{r}_{0}\right)=\frac{I_{\text {inc }}}{\lambda^{2} z_{0}{ }^{2}} G^{2}(a) \int_{S} \frac{\mathrm{d}^{2} r_{i}}{z_{i}^{2}} F\left[K x^{m}\left(\frac{\mathbf{r}_{0}}{z_{0}}+\frac{\mathbf{r}_{i}}{z_{i}}\right)\right] .
$$

When we change to the scaled positions

$$
\mathbf{R}_{0}=K x^{m} \frac{\mathbf{r}_{0}}{z_{0}}
$$

and

$$
\mathbf{W}=-K x^{m} \frac{\mathbf{r}_{i}}{z_{i}}
$$

and use a source with a circular geometry, Eq. (1.6) becomes

$$
I_{0}\left(\mathbf{R}_{0}\right)=\frac{I_{\text {inc }}}{\lambda^{2} z_{0}^{2}} G^{2}(a)\left(K x^{m}\right)^{-2} \int \mathrm{d}^{2} W A_{S}(\mathbf{W}) F\left(\mathbf{R}_{0}-\mathbf{W}\right),
$$

where the aperture function for the source $A_{S}(\mathrm{~W})$ is

$$
A_{S}(\mathbf{W})= \begin{cases}1 & \text { if }|\mathbf{W}| \leq K x^{m} s / z_{i} \\ 0 & \text { if }|\mathbf{W}|>K x^{m} s / z_{i}\end{cases}
$$

Applying the mean-value theorem to Eq. (1.9), we obtain

$$
I_{0}\left(\mathbf{R}_{0}\right) \propto G^{2}(a) \pi s^{2} F_{\text {ave }}\left(\mathbf{R}_{0}\right),
$$

where $F_{\text {ave }}$ is the average value of $F$ resulting from the convolution integral in Eq. (1.9) evaluated at the scaled viewingscreen coordinate $\mathbf{R}_{0}$. As $a$ is increased, the geometric scaling factor $G$ increases, and more rays contribute to the diffraction or scattering. At the same time, however, the interference fringe contrast in $F_{\text {ave }}$ decreases because of the effective blurring produced by the increasingly extended interval of convolution. For small $a$, the increase in $G$ dominates the intensity. For large $a$, the contrast decrease of $F_{\text {ave }}$ dominates. As a result, we expect that, when the diameter of the portion of the target surface pierced by the rays responsible for interference is of the order of $d$, the wave interference reaches its optimum observability. In Section 2 we apply this physical picture to supernumerary rainbows, and in Section 3 we apply it to the glory.

\section{VISIBILITY OF SUPERNUMERARY RAINBOWS}

The primary rainbow occurs when the scattering angle of the rays that are incident upon a water droplet and make one internal reflection within it before exiting possesses a relative minimum at ${ }^{10}$

$$
\theta_{R}=\pi+2 \arcsin \left(\frac{4-n^{2}}{3}\right)^{1 / 2}-4 \arcsin \left(\frac{4-n^{2}}{3 n^{2}}\right)^{1 / 2},
$$

where $n$ is the index of refraction of the water droplet. The rainbow ray that exits the droplet at this minimum scattering angle enters it with an angle of incidence of

$$
\xi_{R}=\arcsin \left(\frac{4-n^{2}}{3}\right)^{1 / 2}
$$

Both the rainbow ray and the incident rays infinitesimally adjacent to it travel the same optical path length

$$
L_{R}=2 a\left[1+3\left(\frac{n^{2}-1}{3}\right)^{1 / 2}\right]
$$

from the plane tangent to the droplet and normal to the entering rainbow ray to the plane tangent to the droplet and normal to the exiting rainbow ray. As a result, the constructive interference or focusing of a finite area of the initial wave front in the direction of the primary rainbow is not affected by the coherence properties of the source.

Pairs of incident rays at various distances to either side of the rainbow ray exit the droplet after one internal reflection with the scattering angle $\theta>\theta_{R}$. They then travel parallel to each other until they reach the position of a distant observer, where they are brought together and interfere with each other constructively, destructively, or at some condition intermediate between these two extremes. ${ }^{11}$ This interference pattern observed on one side of the primary rainbow is the supernumerary rainbow sequence.

In the geometrical ray model for rainbow production, if $\xi$ is the angle of incidence of a light ray upon the droplet and

$$
\xi=\xi_{R}+\epsilon,
$$

then, for small $\epsilon$, the scattering angle of the ray is

$$
\theta=\theta_{R}+\alpha \epsilon^{2}+\beta \epsilon^{3}+\gamma \epsilon^{4}+O\left(\epsilon^{5}\right),
$$

where

$$
\begin{aligned}
& \alpha=\frac{3}{4}\left(\frac{4-n^{2}}{n^{2}-1}\right)^{1 / 2}, \\
& \beta=\frac{n^{2}+8}{16\left(n^{2}-1\right)},
\end{aligned}
$$

and 


$$
\gamma=\frac{17 n^{2}-8}{256\left(n^{2}-1\right)}\left(\frac{4-n^{2}}{n^{2}-1}\right)^{1 / 2} .
$$

Equation (2.5) may be inverted to give

$$
\epsilon_{ \pm}= \pm\left(\frac{\psi}{\alpha}\right)^{1 / 2}-\frac{\beta}{2 \alpha^{2}} \psi \pm \frac{1}{2 \alpha^{5 / 2}}\left(\frac{5}{4} \frac{\beta^{2}}{\alpha}-\gamma\right) \psi^{3 / 2}+O\left(\psi^{2}\right)
$$

for the two geometrical rays $\xi_{+}$and $\xi_{-}$exiting the droplet in the $\theta$ direction parallel to each other, where

$$
\psi=\theta-\theta_{R} \text {. }
$$

The optical path lengths of the $\xi_{ \pm}$rays between the planes tangent to the droplet at their entrance and exit are

$$
\begin{aligned}
L\left(\xi_{ \pm}\right)= & L_{R}+a\left(\frac{4-n^{2}}{3}\right) \psi \pm \frac{a}{2}\left(\frac{4-n^{2}}{3}\right)^{1 / 2}\left(\frac{\psi}{\alpha}\right)^{3 / 2} \\
& +\frac{a}{36}\left(\frac{11 n^{2}-56}{4-n^{2}}\right)\left(\frac{n^{2}-1}{3}\right)^{1 / 2} \psi^{2}+O\left(\psi^{5 / 2}\right) .
\end{aligned}
$$

Constructive interference of the $\xi_{+}$and $\xi_{\text {- rays occurs when }}$ the difference between their path lengths satisfies

$$
L\left(\xi_{+}\right)-L\left(\xi_{-}\right)=N \lambda
$$

for an integer $N \geq 0$, with $N=0$ representing the primary rainbow and $N \geq 1$ representing the supernumerary sequence, or when

$$
\psi=\frac{3}{4}(3)^{1 / 3} \frac{\left(4-n^{2}\right)^{1 / 6}}{\left(n^{2}-1\right)^{1 / 2}}\left(\frac{N \lambda}{a}\right)^{2 / 3} .
$$

The separation $\delta$ of the incident $\xi_{+}$and $\xi_{\text {- rays before they }}$ enter the droplet is

$$
\delta=a \sin \xi_{+}-a \sin \xi_{-} .
$$

For small $\epsilon$ and $\psi$, the separation $\delta$ is proportional to $a^{2 / 3}$.

It was shown recently that the oblateness of falling raindrops causes the observed supernumerary rainbows to be produced by water droplets only of radii near $a \approx 250 \mu \mathrm{m} .{ }^{12,13}$ Using this radius with $\lambda=0.55 \mu \mathrm{m}$ and $n=1.33$ in Eqs. (2.9), (2.13), and (2.14), one obtains the separations of the pairs of incident rays that produce the constructive-interference supernumerary rainbows. These separations are given in Table 1. Since the separations for $N \geq 2$ are beyond the $40-\mu \mathrm{m}$

Table 1. Characteristics of Supernumeraries ${ }^{a}$

\begin{tabular}{rcc}
\hline$N$ & $\delta(\mu \mathrm{m})$ & $V$ \\
\hline 1 & 34.93 & 0.656 \\
2 & 44.25 & 0.491 \\
3 & 50.90 & 0.368 \\
4 & 56.25 & 0.294 \\
5 & 60.83 & 0.235 \\
6 & 64.87 & 0.184 \\
7 & 68.52 & 0.127 \\
8 & 71.86 & 0.059 \\
9 & 73.87 & 0.030 \\
10 & 77.86 & 0.015 \\
\hline
\end{tabular}

$a$ The supernumerary rainbow orders $N$ for a water droplet of radius 0.25 $\mathrm{mm}$, the initial separation $\delta$ of the two incident geometrical rays that produce the supernumerary, and the supernumerary visibility factor defined in Eq. (2.21). For $N \geq 2$, the separation $\delta$ exceeds the nominal spatial coherence width of sunlight. nominal spatial coherence width of sunlight at the Earth, the Sun's incoherence should greatly reduce the visibility of all but the first few supernumeraries.

The extent of the reduction of supernumerary visibility as a result of the finite size of the Sun may be determined quantitatively from Eq. (1.9). As is shown above, the radial extent $\Delta r$ of the portion of the surface of the droplet that is responsible for the constructive interference producing the primary rainbow or each supernumerary rainbow at one location on the viewing screen is proportional to $a^{2 / 3}$. It can also be shown easily that the polar coordinate angular interval $\Delta \phi$ of the portion of the droplet surface that gives constructive interference at one location on the viewing screen is proportional to $a^{-1 / 2}$. Thus the droplet surface area that is responsible for rainbow production at one viewing-screen location is ${ }^{14}$

$$
G=(\Delta r)(r \Delta \phi) \propto a^{7 / 6} .
$$

Using Airy's cubic wave-front model to evaluate the waveinterference factor $F$ in Eq. (1.2), one obtains ${ }^{15}$

$$
F\left(x_{0}, y_{0}\right)=\mathrm{Ai}^{2}\left(-K x^{2 / 3} \frac{y_{0}}{z_{0}}\right) \text {, }
$$

where

$$
K=\left(\frac{2}{3}\right)^{2 / 3} \frac{\left(n^{2}-1\right)^{1 / 2}}{\left(4-n^{2}\right)^{1 / 6}},
$$

where the rectangular coordinates $\left(x_{0}, y_{0}\right)$ are measured with respect to the ray-optics position of the primary rainbow in one localized region of the rainbow arc and where $\mathrm{Ai}$ is the Airy integral, ${ }^{16}$

$$
\operatorname{Ai}(-u)=\left(\frac{3}{2 \pi^{2}}\right)^{1 / 3} \int_{0}^{\infty} \cos \frac{\pi}{2}\left[v^{3}-\left(\frac{12}{\pi^{2}}\right)^{1 / 3} v u\right] \mathrm{d} v .
$$

The locations of the relative maxima of $F$ are determined by the stationary-phase approximation. They are found to be at

$$
\frac{y_{0}}{z_{0}}=\psi \approx \frac{3}{4}(3)^{1 / 3} \frac{\left(4-n^{2}\right)^{1 / 6}}{\left(n^{2}-1\right)^{1 / 2}}\left[\frac{\left(N+\frac{1}{4}\right) \lambda}{a}\right]^{2 / 3}
$$

in this wave-optics model, in agreement with our prior rayoptics prediction of Eq. (2.13). With a single independent radiator serving as the light source, the supernumerary intensity on the viewing screen as a function of the scaled position,

$$
Y_{0}=K x^{2 / 3} y_{0} / z_{0}
$$

is given by Eq. (2.16) and is shown in Fig. 1.

The convolution integral of Eq. (1.9) for the supernumerary rainbow region was calculated as a function of $Y_{0}$ for $G$ and $F$ as given in Eqs. (2.15) and (2.16), respectively. The resulting intensity is shown in Fig. 2. The contrast of the supernumerary rainbows is reduced considerably from that of Fig. 1. The values of the fringe visibility,

$$
V=\frac{I_{\max }-I_{\min }}{I_{\max }+I_{\min }}
$$

for the supernumeraries of Fig. 2 are given in Table 1, for which background light in the supernumerary region was not 


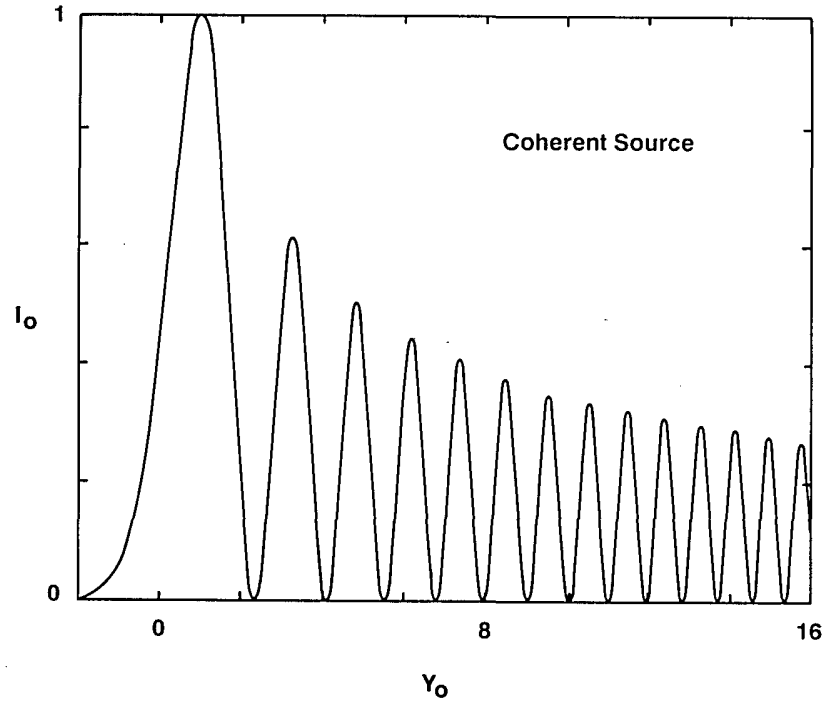

Fig. 1. Intensity in the supernumerary rainbow region of coherent light $(\lambda=0.55 \mu \mathrm{m})$ scattered by a water droplet as a function of the scaled position $Y_{0}$ defined in Eq. (2.20). The primary rainbow intensity is normalized to unity.

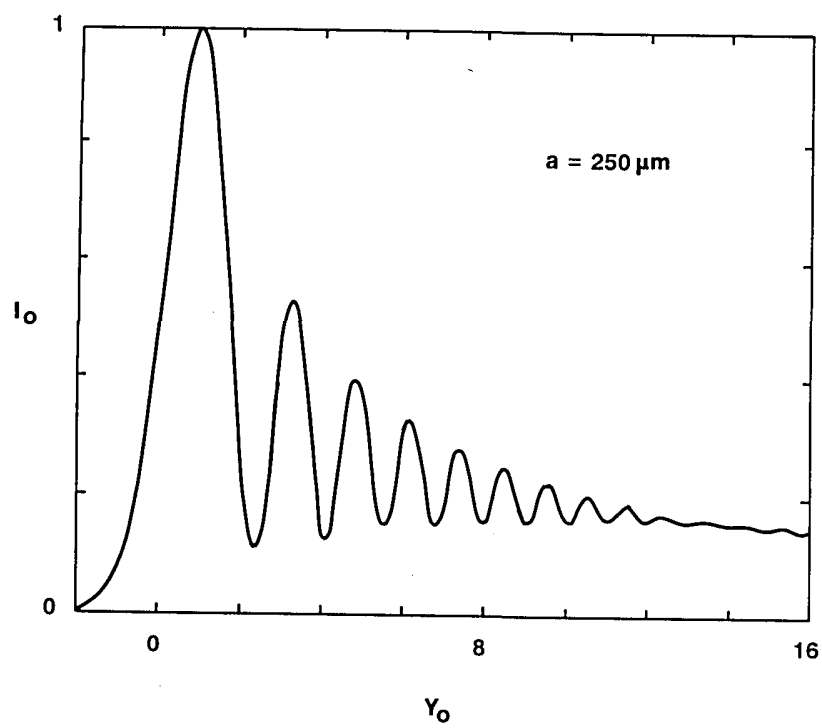

Fig. 2. Intensity in the supernumerary rainbow region of sunlight $(\lambda=0.55 \mu \mathrm{m})$ scattered by a $250-\mu$ m-radius water droplet as a function of the scaled position $Y_{0}$. The primary rainbow intensity is normalized to unity. The difference from Fig. 1 arises from the partial spatial coherence of sunlight.

taken into account. Similar convolution integrals over the solar disk were performed previously. . $^{12,13,17-19}$ However, it appears that a quantitative measure of supernumerary observability that is due to source incoherence was not made previously.

It can be seen from Table 1 that, for $N>3$, visibility decreases to $<0.3$. The way in which fringe visibility translates into human observability depends on the physiological factors mentioned in Section 1. However, in general, decreasing values of $V$ correspond to decreasingly observable situations. Thus the finite coherence width of sunlight at the Earth places a stringent limit on the number of atmospheric supernumerary rainbows that may be observed. In recent years, many beautiful rainbow photographs were published. In almost all of them, only one or two supernumeraries at best are visible, in general agreement with the results in Table 1. Occasionally, as in the photos in Refs. 13 and 20 , as many as three or four supernumeraries can be seen.

In obtaining the results in Table 1 and Figs. 1 and 2, we used the wave-interference model of Eq. (2.16) rather than the exact Mie scattering theory. Mie theory predicts the polarization dependence of the rainbow and also the level of background light upon which the primary rainbow and supernumeraries appear. For a $250-\mu \mathrm{m}$-radius water droplet, the supernumerary-to-background intensity ratio in the dominant polarization is $100: 1$, whereas it is only $5: 1$ for unpolarized light. This background was omitted in our calculations of Eq. (2.21), and physically it is largely removed if the rainbow appears in front of dark clouds or landscape and is observed through a polarizing filter. Our results in Table 1 and Figs. 1 and 2 correspond to this method of observation.

\section{VISIBILITY OF THE GLORY}

Van de Hulst was the first to conjecture that the dominant mechanism in the production of the glory for reasonably small water droplets is the interference of light rays that enter the water droplet at its outer edge, make one internal reflection within it, and exit in the backscattered direction. ${ }^{21}$ The exiting effective toroidal wave front in this model gives rise to the wave-interference factor

$$
F=\left(c_{1}+c_{2}\right)^{2} J_{0}^{2}\left(\frac{x r_{0}}{z_{0}}\right)+\left(c_{1}-c_{2}\right)^{2} J_{2}^{2}\left(\frac{x r_{0}}{z_{0}}\right)
$$

in Eq. (1.2), where $K=1, m=1$, where $c_{1}$ and $c_{2}$ are the products of transmission and internal reflection coefficients of the incident transverse magnetic and transverse electric fields, and where the radial coordinate $r_{0}$ on the viewing screen is measured with respect to the backscattered direction. This wave-interference factor is plotted as a function of the scaled radial coordinate $R_{0}$ in Fig. 3 for ${ }^{22}$

$$
\begin{aligned}
& c_{1}=-0.2, \\
& c_{2}=1.0 .
\end{aligned}
$$

Since waves from the entire circumference of the droplet contribute to glory interference, and since the radial width $\Delta r$ of the edge region of the droplet is proportional to $a^{1 / 3}$, the geometric scaling factor in Eq. (1.2) is ${ }^{23}$

$$
G=2 \pi a(\Delta r) \propto a^{4 / 3}
$$

for glory scattering.

Unfortunately, the index of refraction of water is such that light rays incident at the edge region have a scattering angle of only $\theta=166^{\circ}$ and cannot be backscattered. In order to overcome this difficulty, it was conjectured that surface waves created at the points of incidence, internal reflection, and exit provide the missing $14^{\circ}$ of scattering angle. ${ }^{24}$ Thus the coefficients $c_{1}$ and $c_{2}$ also include the amplitudes for the creation and propagation of surface waves and are functions of the droplet size rather than being strictly constants. ${ }^{25}$ It was proved both experimentally ${ }^{26}$ and numerically ${ }^{26,27}$ that light rays incident at the edge of the droplet are, in fact, a large contributor to the glory interference pattern, and 


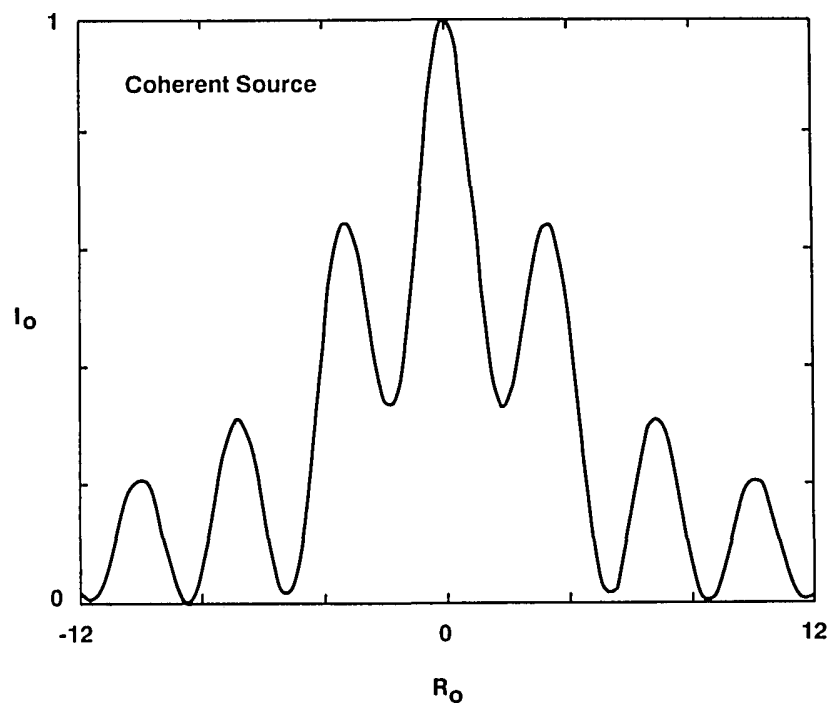

Fig. 3. Intensity in the glory region of coherent light $(\lambda=0.55 \mu \mathrm{m})$ light scattered by a water droplet as a function of the scaled position $R_{0}$ defined in Eqs. (1.7) and (3.1). The backscattered intensity is normalized to unity.

methods for calculating the surface-wave contribution to glory amplitude were devised. .3,28-31 $^{\text {In particular, it was }}$ found that the surface waves propagating along the droplet circumference become increasingly damped as the size of the droplet is increased, and as a result ${ }^{23}$

$$
c_{1} \approx c_{2} \propto \exp \left(-0.4 x^{1 / 3}\right) \text {. }
$$

Even for a perfectly coherent light source, surface-wave damping places a limit on the size of water droplets that can produce glories by the van de Hulst mechanism. First, the droplet must be large enough that the geometrical ray model for backscattering is reasonably accurate. This occurs for $x$ $\gtrsim 100$. Second, since the contribution of the van de Hulst term to the glory intensity is proportional to ${ }^{23}$

$$
I_{0} \propto x^{8 / 3} \exp \left(-0.8 x^{1 / 3}\right),
$$

the intensity produced by this mechanism reaches a relative maximum at

$$
x=1000
$$

or

$$
a \approx 88 \mu m .
$$

For increasing $a$ below this maximum, the increase in the participating portion of the surface area of the droplet parameterized by the geometric scaling factor causes more light rays to contribute to glory scattering. As a result, in this regime the glory appears brighter for increasing $a$. Above this maximum, the damping of the surface waves reduces the one-internal-reflection contribution to the glory for increasing $a$. For particle radii larger than $88 \mu \mathrm{m}$, the structure of the backscattered intensity becomes complicated. At these large particle sizes, the 10th-order rainbow contribution to the glory potentially dominates. However, since the 10th-order rainbow extends over an angular interval of more than $12.6^{\circ}$ for the visible spectrum,,$^{10}$ its contribution to backscattering will be much less important for some wavelengths than for others. In addition, the amplitude for backscattered axial rays also increases slowly with particle size. ${ }^{32}$ However, when the diameter of the droplet exceeds the coherence width of sunlight, the light rays entering at the right-hand edge of the droplet are incoherent with the light rays entering at the left-hand edge. As a result, the coherence width of sunlight at the Earth should decrease the contrast of the glory interference pattern for $a \gtrsim 20 \mu \mathrm{m}$. This limiting radius is above the $x \approx 100$ limit for the applicability of the geometrical ray model, and it is more restrictive than the surface-wave damping limit. In this smalldroplet-size regime, the 10th-order rainbow contribution is almost an order of magnitude smaller than the van de Hulst term and need not be considered.

In order to verify that the source coherence width limit is indeed more restrictive, the glory intensity for the extended source of Eq. (1.9) was calculated as a function of $a$ and $R_{0}$ by using Eqs. (3.1) and (3.3) with

$$
\begin{aligned}
& c_{1}=-0.2 \exp \left(-0.4 x^{1 / 3}\right), \\
& c_{2}=1.0 \exp \left(-0.4 x^{1 / 3}\right) .
\end{aligned}
$$

The amplitudes of these coefficients are the estimates given by van de Hulst, ${ }^{22}$ and the exponential damping factor is the value derived by Khare and Nussenzveig. ${ }^{23}$ In actuality, the amplitudes as well are size dependent, but we decided to use the constants of Ref. 22, since the angular dependence of the glory pattern is only weakly dependent on the specific values of $c_{1}$ and $c_{2}$. As a measure of fringe observability, we chose to examine the quantity

$$
U=x^{8 / 3} \exp \left(-0.8 x^{1 / 3}\right) V
$$

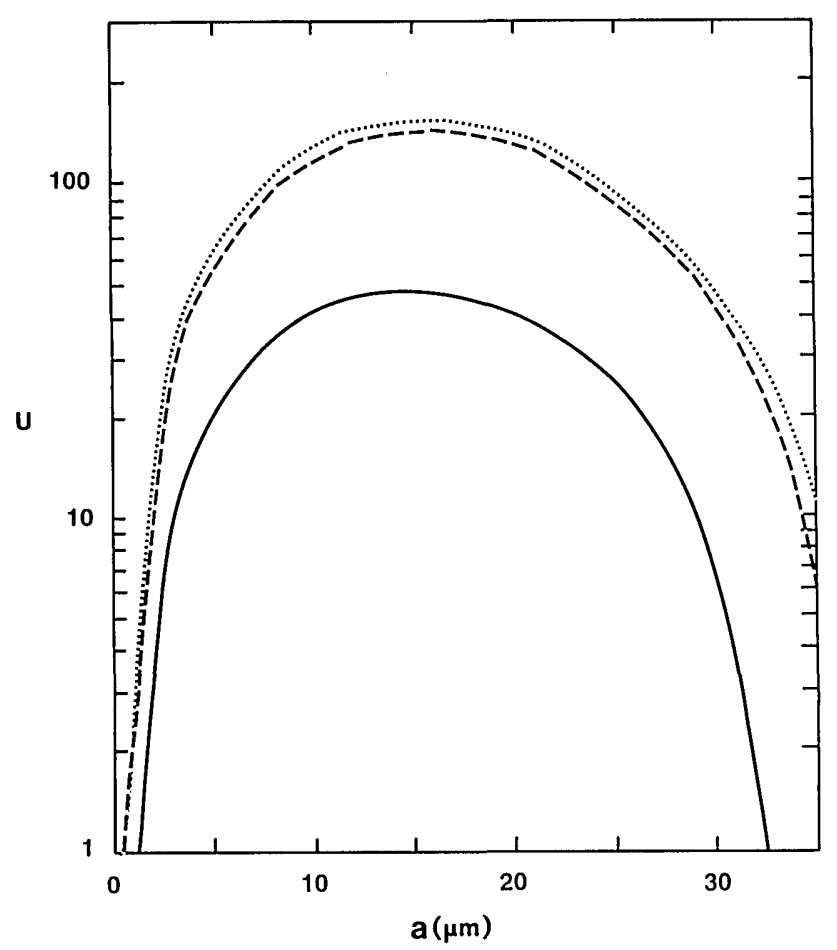

Fig. 4. Glory observability factor $U$ of Eq. (3.10) for the first glory ring (solid curve), the second glory ring (dashed curve), and the third glory ring (dotted curve) as a function of the water droplet radius. 


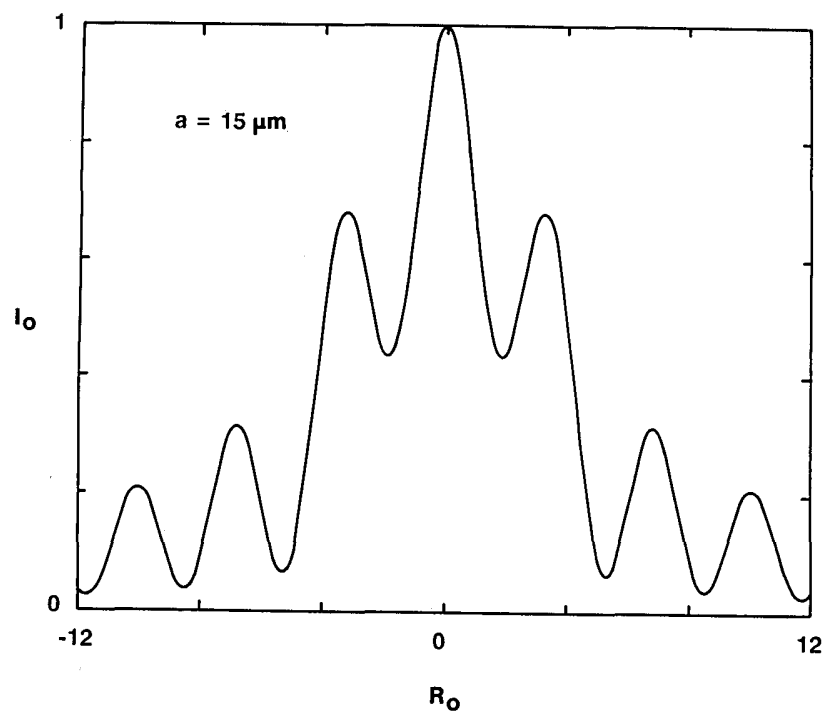

Fig. 5. Intensity in the glory region of sunlight $(\lambda=0.55 \mu \mathrm{m})$ scattered by a $15-\mu \mathrm{m}$-radius water droplet as a function of the scaled position $R_{0}$. The backscattered intensity is normalized to unity. The difference from Fig. 3 arises from the partial spatial coherence of sunlight.

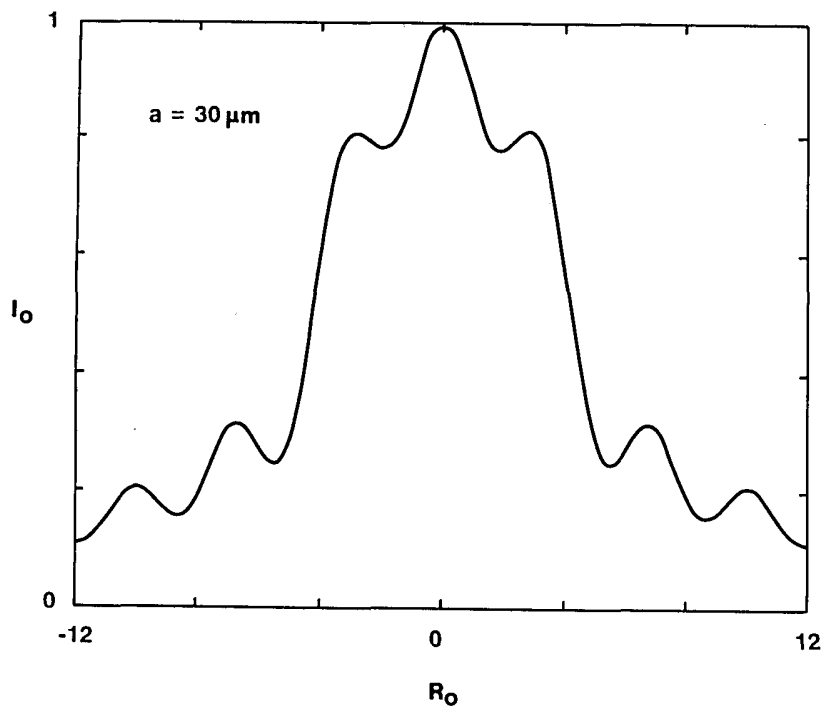

Fig. 6. Intensity in the glory region of sunlight $(\lambda=0.55 \mu \mathrm{m})$ scattered by a $30-\mu \mathrm{m}$-radius water droplet as a function of the scaled position $R_{0}$. The backscattered intensity is normalized to unity. The difference from Fig. 3 arises from the partial spatial coherence of sunlight.

where $V$ is the fringe visibility of Eq. (2.21). It was calculated for this situation from the result of the convolution integral of Eqs. (1.9), (3.1), and (3.3). This quantity is by no means a unique measure of interference-fringe observability, but it does include the important physical factors that influence observability. In Fig. $4, U$ is plotted as a function of the droplet radius for the first three glory rings. Again, the background intensity that is due to backscattered axial rays and high-order rainbows was not included. As is seen in this figure, $U$ attains a relative maximum for $a \approx 15 \mu \mathrm{m}$, in agreement with our simple picture of the coherence width limitation of glory visibility. The intensity of the glory rings as a function of the scaled position $R_{0}$, calculated by using Eqs. (1.9), (3.1), and (3.3), is shown in Figs. 5 and 6 for a 15$\mu \mathrm{m}$-radius droplet and for a $30-\mu \mathrm{m}$-radius droplet, respectively. The first droplet size is at the relative maximum of $U$, and the second is somewhat beyond it. This suggests that, as is the case for supernumerary rainbows, ${ }^{12,18}$ in a wide size distribution of water droplets the glory is produced primarily by a small range of droplet radii.

When the unpolarized Mie scattered intensity is calculated as a function of the scattering angle near the backscattered direction for $a \approx 15 \mu \mathrm{m}$, the resulting intensity deviates substantially from Fig. 3 in two respects. First, the glory interference pattern is superposed upon a background light intensity. The interference maximum-to-background intensity ratio varies between approximately $3: 1$ and 10:1. Second, the scattered intensity at a scattering angle of $180^{\circ}$ can be much larger or smaller than the prediction of Fig. 3, depending on whether the droplet diameter is at a scattering resonance or away from one. ${ }^{33}$ In any event, the angular separation between the various Mie scattering maxima and minima is the same as the angular separation shown in Fig. 3. As a result, a convolution of the Mie intensity would decrease the contrast between these maxima and minima to the same extent that they were decreased in Fig. 4. Although the numerical values of $U$ would be different for the convolved Mie intensity from what they were in Fig. 4, they would peak at roughly the same droplet radii, and our conclusion about the coherence width limitation to glory observability would be the same.

The average radii of the water droplets producing the glories analyzed by van de Hulst were $14 \mu \mathrm{m}$, and the radii of the droplets producing the glory described by Bryant and Jarmie $^{34}$ were $9 \mu \mathrm{m}$. It is of interest to compare these values and the optimum droplet radii of Fig. 4 with the most probable radius of water droplets found in clouds and mists. The most probable cloud droplet radius depends to some extent on the cloud type and the location of the droplet within the cloud. However, many measurements place the most probable radius in the range of $5-12 \mu \mathrm{m} .{ }^{35,36}$ Similar radii occur for fog particles. ${ }^{37,38}$ It is a fortunate accident that the optimum droplet radii for glory production are so close to the most probable radius of cloud droplets. If the water droplets in clouds were a factor of 4 larger or smaller, the observation of a naturally occurring glory would be a rare occurrence indeed.

\section{REFERENCES}

1. K. Sassen, "Angular scattering and rainbow formation in pendant drops," J. Opt. Soc. Am. 69, 1083-1089 (1979).

2. P. L. Marston, "Rainbow phenomena and the detection of nonsphericity in drops," Appl. Opt. 19, 680-685 (1980).

3. D. S. Langley and P. L. Marston, "Glory in optical backscattering from air bubbles," Phys. Rev. Lett. 47, 913-916 (1981).

4. W. P. Arnott and P. L. Marston, "Optical glory of small freely rising gas bubbles in water: observed and computed crosspolarized backscattering patterns," J. Opt. Soc. Am. A 5, 496506 (1988).

5. W. D. Wright, The Measurement of Colour (Hilger, London, 1969), Chap. 4, App. 2, Table 4.

6. J. A. Prins and J. J. M. Reesnick, "Buigingstheorie en trichromatische specificatie van de regenboogkleuren," Physica 11, 4960 (1944).

7. E. Hecht, Optics, 2nd ed. (Addison-Wesley, Reading, Mass., 1987), Secs. 12.1, 12.4.1. 
8. R. W. Wood, Physical Optics, 3rd. ed. (Macmillan, New York, 1934), pp. 186-187.

9. J. W. Goodman, Introduction to Fourier Optics (McGraw-Hill, New York, 1968), Sec. 6-3.

10. J.D. Walker, "Multiple rainbows from single drops of water and other liquids," Am. J. Phys. 44, 421-433 (1976).

11. J. D. Walker, "Mysteries of rainbows, notably their rare supernumerary arcs," Sci. Am. 242 (6), 174-184 (1980).

12. A. B. Fraser,"Why can the supernumerary bows be seen in a rain shower?" J. Opt. Soc. Am. 73, 1626-1628 (1983).

13. A. B. Fraser, "Chasing rainbows," Weatherwise 36, 280-289 (1983).

14. R. A. R. Tricker, Introduction to Meteorological Optics (Elsevier, New York, 1970), p. 181

15. H. C. van de Hulst, Light Scattering by Small Particles (Dover, New York, 1981), Sec. 13.23.

16. M. Abramowitz and I. A. Stegun, Handbook of Mathematical Functions (National Bureau of Standards, Washington, D.C., 1964), Art. 10.4.32.

17. C. W. Querfeld, "Mie atmospheric optics," J. Opt. Soc. Am. 55, 105-106 (1965).

18. G. P. Können, "Appearance of supernumeraries of the secondary rainbow in rain showers," J. Opt. Soc. Am. A 4, 810-816 (1987).

19. S. D. Gedzelman, "Rainbows in strong vertical atmospheric electric fields," J. Opt. Soc. Am. A 5, 1717-1721 (1988).

20. D. Falk, D. Brill, and D. Stork, Seeing the Light (Harper \& Row, New York, 1986), color plate 2.3.

21. H. C. van de Hulst, "A theory of the anti-coronae," J. Opt. Soc. Am. 37, 16-22 (1947).

22. See Ref. 15, Sec. 13.33 .

23. V. Khare and H. M. Nussenzveig, "The theory of the glory," in U. Landman, ed., Statistical Mechanics and Statistical Methods in Theory and Application (Plenum, New York, 1977), Sec. 8.3, pp. 723-765.

24. See Ref. 15, Secs. 13.32, 17.42 .
25. J. V. Dave, "Scattering of visible light by large water spheres," Appl. Opt. 8, 155-164 (1969).

26. T. S. Fahlen and H. C. Bryant, "Optical back scattering from single water droplets," J. Opt. Soc. Ám. 58, 304-310 (1968).

27. H. C. Bryant and A. J. Cox, "Mie theory and the glory," J. Opt. Soc. Am. 56, 1529-1532 (1966).

28. H. M. Nussenzveig, "High-frequency scattering by a transparent sphere. I. Direct reflection and transmission," J. Math. Phys. 10, 82-124 (1969).

29. H. M. Nussenzveig, "High-frequency scattering by a transparent sphere. II. Theory of the rainbow and the glory," J. Math. Phys. 10, 125-176 (1969).

30. V. Khare and H. M. Nussenzveig, "Theory of the glory," Phys. Rev. Lett. 38, 1279-1282 (1977).

31. H. M. Nussenzveig, "Complex angular momentum theory of the rainbow and the glory," J. Opt. Soc. Am. 69, 1068-1079, 11931194 (1979).

32. H. Inada and M. A. Plonus, "The geometric optics contribution to the scattering from a large dense dielectric sphere," IEEE Trans. Antennas Propag. AP-18, 89-99 (1970).

33. J. R. Probert-Jones, "Resonance component of backscattering by large dielectric spheres," J. Opt. Soc. Am. A 1, 822-830 (1984).

34. H. C. Bryant and N. Jarmie, "The glory," Sci. Am. 231(7), 60-71 (1974), Fig. 2.

35. B. J. Mason, The Physics of Clouds (Clarendon, Oxford, 1971), Fig. 3.9.

36. T. A. Cerni, "Determination of the size and concentration of cloud drops with an FSSP," J. Clim. Appl. Meteor. 22, 13461355 (1983).

37. E. J. McCartney, Optics of the Atmosphere (Wiley, New York, 1976), Fig. 3.19.

38. R. G. Pinnick, D. M. Garvey, and L. D. Duncan, "Calibration of Knollenberg FSSP light-scattering counters for measurement of cloud droplets," J. Appl. Meteorol. 20, 1049-1057 (1981). 